\title{
El aprendizaje cooperativo, educación desde la participación social en estudiantes de bachillerato
}

Fecha de recepción : 10 de febrero de 2020 • Fecha de aceptación: 8 de abril de 2020

\author{
Fecha de aceptación: 8 de abril de 2020
}

- Fecha de publicación: 10 de mayo de 2020

\author{
Wilmer Rubén Paredes León ${ }^{1}$ \\ Pontificia Universidad Católica del Ecuador \\ willeon25@hotmail.com \\ https://orcid.org/0000-0003-2293-4050
}

Gerardo Ramos Serpa²

Universidad Regional Autónoma de Los Andes

gramosserpa@gmail.com

https://orcid.org/0000-0003-3172-555X

\section{Resumen}

El aprendizaje cooperativo ha venido cumpliendo una tarea muy necesaria e importante en el quehacer educativo, pues se constituye como una herramienta muy útil; sin embargo, este tipo de aprendizaje ha sido utilizado como secundario, poco común y en ocasiones, etiquetado como un elemento innecesario en el proceso de enseñanza. Por lo descrito, el objetivo de la investigación es diagnosticar el estado de la formación cooperativa en el nivel de bachillerato y su relación directa con la participación social. La estudio posee un enfoque mixto con un diseño explicativo secuencial, mediante una triangulación de investigación a través de un cuestionario, una entrevista y una guía de observación, entre los resultados principales se encuentra que en las asignaturas de Lengua-Literatura y Matemática se aplica con menor medida el aprendizaje cooperativo, mientras que en Ciencias Naturales y Ciencias Sociales se da mayor apertura a este tipo de instrucción. Finalmente, se concluye que los docentes concuerdan que el trabajo de este tipo está basado en la formación de equipos 
de aprendizaje que, a su vez, desarrollan habilidades sociales mediante la realización de actividades con ejemplos pertinentes y la guía del docente.

Palabras clave: aprendizaje cooperativo, educación, docentes, habilidades sociales, enseñanza.

\begin{abstract}
Cooperative learning has been fulfilling a very necessary and important task in the educational endeavor, it constitutes a very useful tool, however, this type of learning has been used as a secondary, rare and sometimes labeled as an element unnecessary in the teaching process. Based on what has been described, the objective of the research is to diagnose the state of cooperative learning at the high school level and its direct relationship with social participation. The study has a mixed approach and with a sequential explanatory design, through a triangulation of research through a questionnaire, an interview and an observation guide, among the main results of this research is that in the subjects of Language-Literature and Mathematics is applied to a lesser extent cooperative learning, while in Natural Sciences and Social Sciences are the subjects that give more openness to this type of instruction. Finally, it is concluded that the teachers agree that cooperative work is based on the formation of learning teams that in turn develop social skills by carrying out activities with relevant examples and the teacher's guide.
\end{abstract}

Keywords: cooperative learning, education, teacher, social skills, teaching. 


\section{Introducción}

A pesar que la evolución y la globalización han tomado control y posición importante en el ámbito educativo, no se puede negar que todavía prevalece ese paradigma tan marcado del tradicionalismo, caracterizado por el fomento del trabajo individual en el proceso de aprendizaje, la pasividad, conformismo, la idea homogénea, estrategias y técnicas marcadas y rigurosas, donde todos los estudiantes encajan en solo molde.

Un tema que ha causado controversia en la educación a nivel mundial es la inclusión, y se ha logrado vincularla a este sistema gracias a la aplicación de la estrategia innovadora de aprendizaje cooperativo, así rescata esta idea la Organización de las Naciones Unidas para la Educación, la Ciencia y la Cultura (UNESCO) (2011) al afirmar que la educación debe ser totalmente incluyente, con un enfoque pedagógico centrado en los estudiantes y basado en la cooperación tanto en los maestros y maestras en la enseñanza, como en los y las estudiantes en el aprendizaje y señala también que este es el medio más eficaz para lograr una educación integral para todos.

De la misma manera, basado en elementos globales e imprescindibles, el Ministerio de Educación de Ecuador (MINEDUC) (2016) en el currículo de los niveles de educación obligatoria en el apartado del perfil de salida del bachiller ecuatoriano en el valor fundamental de la solidaridad establece una conexión muy estrecha con el aprendizaje cooperativo al considerar que los alumnos en su quehacer educativo deben aprender, ensayar, trabajar y convertir este valor en un principio fundamental. Los aspectos donde converge este tipo de instrucción con la solidaridad son:

S.1. Asumimos responsabilidad social y tenemos capacidad de interactuar con grupos heterogéneos, procediendo con comprensión, empatía y tolerancia.

S.4. Nos adaptamos a las exigencias de un trabajo en equipo en el que comprendemos la realidad circundante y respetamos las ideas y aportes de las demás personas (MINEDUC, 2016, p. 8).

Al observar una educación conservadora y tradicionalista cada vez son más las ideas y las voces que se manifiestan con el objetivo de cambiar la concepción educativa, y una de esas formas y estrategias es el aprendizaje cooperativo, como lo dan a conocer López y Acuña (2018) al decir que esta estrategia permite a los estudiantes no solo aprender el material de la clase sino a su vez ayudar a los compañeros de su equipo a que juntos aprendan y cumplan los objetivos. Coinciden con esta idea Doporto y Rodríguez (2015) y añaden que favorece a los estudiantes a desarrollar destrezas y conocimientos, pero enriquecidos por las diversas experiencias y contextos.

El aprendizaje cooperativo como recurso integrador requiere la inmersión de los agentes educativos, así como de las técnicas y recursos necesarios que sirvan como apoyo y sustento para cumplir con este fin. Román y De la Torre (2017) dan a conocer que este aprendizaje es una metodología educativa innovadora, con enfoque constructivista, incluyente y con el potencial suficiente para modificar las prácticas pedagógicas en las aulas. 
Al establecer una conexión con las teorías y pensamientos educativos clásicos y su relación directa con el aprendizaje cooperativo, Vygotsky (1979) mantiene que la zona de desarrollo próximo no es otra cosa que esa distancia real de progreso que se marca en el niño en su capacidad de resolver independientemente un problema y el nivel de crecimiento que se potencia en la resolución del mismo, con la guía de un adulto o también por la colaboración de otro compañero más competente. La cooperación favorece el progreso, porque es posible que los niños de edades parejas operen dentro de las zonas de desenvolvimiento próximo de los demás, al participar como modelos de comportamientos más avanzados en sus propios grupos.

Para describir la influencia de la cooperación sobre el aprendizaje, Vygotsky (1978) expresa que las funciones se establecen primero en lo colectivo, como relaciones entre niños y así después se transforman en funciones psíquicas de la persona. Así también Bell y Perret (1985) señalan que las investigaciones han confirmado que cuando líderes y no líderes de la misma edad trabajan en cooperación en actividades que requieren conservación, los no líderes suelen desarrollar y mantener conceptos de conservación.

Es normal pensar que un aprendizaje tradicional tiene mucha más validez y confianza que un aprendizaje cooperativo por el hecho de que ha sido el aprendizaje que más se ha utilizado en la educación, pero ante eso Slavin y Johnson (1999) contraponen esta ideología y lo hacen al exponer sus teorías motivacionales que se centran en las recompensas u objetivos del equipo; Deutsch (1949) identifica tres estructuras de objetivos: cooperativas, en las que los esfuerzos de cada individuo aportan a los logros de los otros; competitivas, en las que los esfuerzos de cada persona buscan frustrar los objetivos de los demás, e individualistas, en las que los esfuerzos individuales no tienen efectos sobre los objetivos ajenos.

Los teóricos de este tipo de formación en la línea motivacional confrontan al aprendizaje tradicional en el hecho de que este crea normas, separación, distinción entre los estudiantes, así lo manifiesta Coleman (1961) al alejarse del aprendizaje tradicional pues considera que este se opone a los esfuerzos académicos y crea conflictos entre los propios pares. Al realizar algunas investigaciones se ha comprobado que cuando los alumnos trabajan juntos por un objetivo común, estos llegan a establecer normas que favorecen al equipo haciendo lo que sea necesario para obtener grandes resultados, así lo pone de manifiesto Deutsch (1949). A esta idea se une Hulten y De Vries (1976) al confirmar, que, en un aula cooperativa, un estudiante que pone empeño y dedicación, asiste a clases de manera regular y ayuda a sus compañeros a aprender, recibe aplausos y el aliento de su equipo, la cual diferencia acentuadamente con lo que ocurre en una clase tradicional.

Es necesario establecer un enfoque en el aporte que brinda el aprendizaje cooperativo en el desarrollo de la participación social, considerado como un eje primordial en la formación de un ser humano.

Para que este aprendizaje resulte favorable es primordial tomar en cuenta cinco elementos esenciales, que, según Johnson, Johnson y Holubec (1999) son: (1) interdependencia positiva, los estudiantes interactúan entre todos y todos dependen de todos para así lograr los objetivos planteados: (2) la responsabilidad individual, cada miembro del equipo es responsable del aporte que debe dar para de esta manera contribuir al progreso de su equipo, (3) la interacción 
promotora, los integrantes del equipo deben estar en contacto directo motivándose mutuamente, (4) procesamiento grupal, los estudiantes del equipo deben trabajar por un bien común, respaldarse, tomar decisiones de manera consensuada, (5) habilidades sociales, los estudiantes aprenden a respetar, a escuchar, a motivar, a respetar, a ser tolerante.

Las habilidades sociales mantienen una estrecha relación con el aprendizaje cooperativo y con los cinco elementos esenciales que la componen. Para Berbena, Sierra y Vivero (2017) son conductas que se observan, aprenden y que se ponen en práctica en el intercambio social.

El papel del docente en el aprendizaje cooperativo juega un rol primordial, pues es la persona que enseña, guía, forma, fomenta y brinda las herramientas necesarias para trabajar de manera cooperativa. El trabajo del docente en el aprendizaje cooperativo se da en tres fases: (1) planificación, el docente prepara las actividades que desarrollará, de acuerdo al contexto, (2) desarrollo, supone la puesta en práctica de lo planificado, (3) análisis y reflexión, el docente en base a lo realizado analiza, valora, emite juicios de valor y se prepara para cambios a futuro (Martín., Martín, J., y Mayo, 2014).

Para complementar la idea del rol del docente frente al aprendizaje cooperativo Doporto y Rodríguez (2015) explica que el docente debe aprender a planificar, argumentar, debatir, decidir, organizar, dar protagonismo a este aprendizaje, y añade que la cooperación es necesaria en edades de bachillerato, tanto en lo que se refiere al desarrollo personal y convivencia como para el ejercicio profesional.

A su vez, Seijo, López, C., López, M. y Virseda (2015) sostienen que a los profesores les corresponde adoptar planes de mejora, modificar programas y currículos educativos, con el afán de brindar procesos educativos disruptivos que potencialicen la educación y las relaciones sociales entre estudiantes.

Gómez y Gómez (2016) exponen que el aprendizaje cooperativo tiene una serie de ventajas para el estudiante tales como: promover las relaciones sociales, la interrelación entre compañeros y docentes, la creatividad, la responsabilidad y autonomía, y en definitiva los prepara para la vida.

Mientras que, Cifuentes y Meseguer (2015), añade a estos beneficios que el trabajar en equipo es el medio apto para desarrollar aprendizajes por competencias, es decir en este caso, al aplicar este aprendizaje, los estudiantes de bachillerato se encuentran preparados para ingresar a la educación superior, el objetivo de este estudio es diagnosticar el estado del aprendizaje cooperativo en el subnivel de bachillerato general unificado de la Unidad Educativa "Suizo".

\section{Metodología}

La propuesta metodológica que propone Pinos, Ayala y Bonilla (2019) para que los estudiantes desarrollen las competencias, es por medio de situaciones didácticas que generen un ambiente creativo en las aulas, considerando que el aprendizaje no es un proceso receptivo, sino activo de elaboración de significados, que es más efectivo cuando se desarrolla con la interacción con otras personas, al compartir e intercambiar información y solucionar problemas colectivamente. 
Para esta investigación se utilizó un enfoque mixto, debido a incorporan un conjunto de procesos críticos, empíricos y sistemáticos, lo cual involucra la recolección y el análisis de datos cuantitativos y cualitativos (Hernández Sampieri y Fernández Collao, 2016). El estudio cuenta con un diseño explicativo secuencial considerando que:

El diseño se caracteriza por una primera etapa en la cual se recaban y analizan datos cuantitativos, seguida de otra donde se recogen y evalúan datos cualitativos. La mezcla mixta ocurre cuando los resultados cuantitativos iniciales informan a la recolección de los datos cualitativos. Cabe señalar que la segunda fase se construye sobre los resultados de la primera. Finalmente, los descubrimientos de ambas etapas se integran en la interpretación y elaboración del reporte del estudio (Hernández - Sampieri, Fernández, \& Baptista, 2014, p.554).

Se trabajó con una población de 69 estudiantes y 4 docentes pertenecientes a los tres niveles del bachillerato general unificado (BGU) de la "Unidad Educativa Suizo" a los primeros se les aplicó el Cuestionario de Aprendizaje Cooperativo en Clase (CACCL), basado en el cuestionario inicial de Fernández, Cecchini, Méndez., G Méndez., A, y Prieto (2017), instrumento que cuenta con un nivel de confiabilidad del 0,91, resultado obtenido del Alpha de Crombach; el mismo que evalúa cinco sub-escalas: Habilidades sociales, Procesamiento grupal, Interdependencia positiva, Interacción promotora y Responsabilidad individual. Y para los segundos, se elaboró una guía de observación para ser aplicada a los docentes del BGU, que cuenta con seis indicadores como: organización y estructuración de los equipos de trabajo, claridad de las normas y forma de trabajar, relación interpersonal, rol del maestro, control de los equipos de trabajo y realización de las actividades

Se realizó un análisis de los tres años del BGU en donde se obtuvo, la media, intervalo de confianza, mediana, varianza, desviación estándar, mínimo, máximo, rango, asimetría y curtosis; estos datos fueron procesados con el programa SPSS, luego se realiza un diagrama comparativo de los tres años del BGU de cajas y bigotes del aprendizaje cooperativo que permite una visualización de los tres grupos para observar si existen diferencias significativas. Finalmente, como los datos no cumplieron el supuesto de normalidad se realiza una correlación de las dimensiones que conforman el CCACL usando el coeficiente de correlación Spearman.

La información obtenida de las áreas de Lengua y Literatura, Matemática, Ciencias Sociales y Ciencias Naturales se procesó a través de NVIVO 10, encargado de realizar un análisis cualitativo, clasificando, ordenando y organizando la información que se obtuvo en el levantamiento de datos, con el fin de obtener la relación de los datos ingresados.

\section{Resultados}

El diagnóstico del aprendizaje cooperativo en el Bachillerato General Unificado tiene dos enfoques, el primero relacionado con la evaluación hecha (mediante una guía de observación) a los docentes. En esta parte se analiza el grado en el que se aplica el aprendizaje cooperativo en el aula de clases. Esto, en función de 7 indicadores a los que se les compara de acuerdo al año y a la asignatura, ver Tabla 1. 
Tabla 1.

Aplicación de aprendizaje cooperativo comparado por año

\begin{tabular}{|c|c|c|c|c|c|c|c|c|}
\hline $\begin{array}{l}\frac{\pi}{\mathscr{2}} \\
\frac{\pi}{\pi} \\
\frac{0}{0} \\
\frac{0}{0}\end{array}$ & 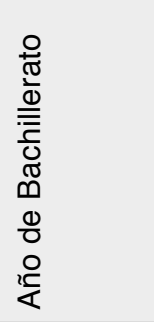 & 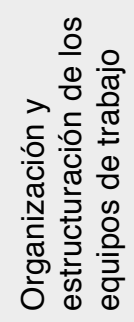 & 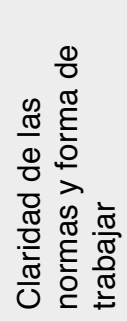 & 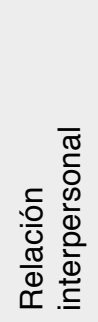 & 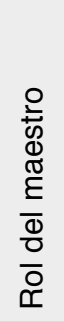 & 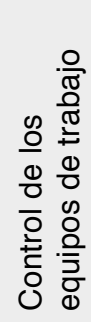 & 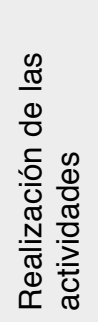 & 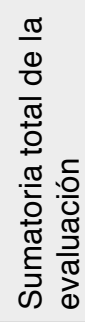 \\
\hline LENGUA & Segundo & 22 & 12 & 12 & 36 & 16 & 11 & 109 \\
\hline MATEMAT. & Primero & 14 & 12 & 14 & 29 & 9 & 12 & 90 \\
\hline EE.SS. & Segundo & 21 & 15 & 18 & 46 & 19 & 16 & 135 \\
\hline CC.NN. & Tercero & 21 & 15 & 19 & 45 & 17 & 13 & 130 \\
\hline
\end{tabular}

Fuente: elaboración propia del cuestionario de aprendizaje cooperativo en clase

Los resultados que se presentan en la tabla revelan que el aprendizaje cooperativo se aplica en diferente medida con respecto a la asignatura, donde matemáticas y lengua son las materias en las que menos se emplea, mientras que las ciencias sociales y naturales son las que mayor apertura dan a esta estrategia didáctica. Esta diferencia se acentúa en la relación interpersonal, el rol del maestro y la realización de actividades.

Las tablas, a continuación, muestran el segundo enfoque, basado en un análisis exploratorio realizado con el fin de determinar, en base a la percepción de los alumnos, el nivel de aplicación del aprendizaje cooperativo en las aulas de clase.

Tabla 2.

Análisis exploratorio - Nivel de aplicación de A.C. $1^{\circ} \mathrm{BGU}$

\begin{tabular}{lll}
\hline Primero BGU & & Estadístico \\
\hline & Media & 76,5000 \\
\cline { 2 - 3 } $\begin{array}{l}\text { 95\% de intervalo de } \\
\text { confianza para la } \\
\text { media }\end{array}$ & $\begin{array}{l}\text { Límite } \\
\text { inferior }\end{array}$ & 71,5087 \\
\cline { 2 - 3 } & $\begin{array}{l}\text { Límite } \\
\text { superior }\end{array}$ & 81,4913 \\
\cline { 2 - 3 } Mediana & 79,0000 \\
\hline Varianza & 113,737 \\
\cline { 2 - 3 } Desv. Desviación & 10,66475 \\
\cline { 2 - 3 } & Mínimo & 46,00 \\
\hline Máximo & 90,00 \\
\cline { 2 - 3 } Rango & 44,00 \\
\cline { 2 - 3 } & Asimetría & $-1,266$ \\
\hline & Curtosis & 2,201 \\
\hline
\end{tabular}

Fuente: elaboración propia en base al cuestionario de aprendizaje cooperativo en clase

Nota. Análisis exploratorio de la percepción de los alumnos del primer año del bachillerato general unificado sobre la aplicación del aprendizaje cooperativo. 
En la Tabla 2 los datos se distribuyen de manera leptocúrtica, con asimetría hacia la izquierda y desviación típica de 10,66. La suma total de las valoraciones otorgadas por los estudiantes, en cada ítem, varía en un rango de 44, siendo 46 la puntuación más baja y 90 la más alta. Esto permite afirmar que el nivel de aplicación del aprendizaje cooperativo tiene una tendencia marcada de mediana igual a 79 y una media muestral de 76,50 que varía dentro del intervalo: $[71,50 ; 81,49]$.

Tabla 3.

Análisis exploratorio - Nivel de aplicación de A.C. $2^{\circ}$ BGU

\begin{tabular}{lll}
\hline Segundo BGU & Estadístico \\
\hline Media & 73,6154 \\
\hline $\begin{array}{l}\text { 95\% de intervalo de } \\
\text { confianza para la } \\
\text { media }\end{array}$ & Límite inferior & 69,9134 \\
\cline { 2 - 3 } Mediana & Límite superior & 77,3174 \\
\hline Varianza & 75,0000 \\
\hline Desv. Desviación & 84,006 \\
\hline Mínimo & 9,16549 \\
\hline Máximo & 53,00 \\
\hline Rango & 86,00 \\
\hline Asimetría & 33,00 \\
\hline Curtosis &,- 706 \\
\hline
\end{tabular}

Fuente: elaboración propia en base al cuestionario de aprendizaje cooperativo en clase Nota. Análisis exploratorio de la percepción de los alumnos del segundo año del bachillerato general unificado sobre la aplicación del aprendizaje cooperativo.

Los datos se distribuyen de manera platicúrtica, con asimetría hacia la izquierda y desviación típica de 9,17. En la Tabla 3 la suma total de las valoraciones otorgadas por los estudiantes, en cada ítem, varía en un rango de 33, siendo 53 la puntuación más baja y 86 la más alta. Esto permite afirmar que el nivel de aplicación del aprendizaje cooperativo tiene una tendencia no muy marcada de mediana igual a 75 y una media muestral de 73,62 que varía dentro del intervalo: $[69,91 ; 77,31]$. 
Tabla 4.

Análisis exploratorio - Nivel de aplicación de A.C. $3^{\circ}$ BGU

\begin{tabular}{|c|c|c|c|}
\hline \multicolumn{3}{|l|}{ Tercero BGU } & \multirow{2}{*}{$\begin{array}{l}\text { Estadístico } \\
72,9565\end{array}$} \\
\hline & Media & & \\
\hline & $95 \%$ de intervalo & Límite inferior & 68,8822 \\
\hline & $\begin{array}{l}\text { de confianza para } \\
\text { la media }\end{array}$ & Límite superior & 77,0308 \\
\hline & Mediana & & 72,0000 \\
\hline & Varianza & & 88,771 \\
\hline & Desv. Desviación & & 9,42182 \\
\hline & Mínimo & & 57,00 \\
\hline & Máximo & & 87,00 \\
\hline & Rango & & 30,00 \\
\hline & Asimetría & &,- 130 \\
\hline & Curtosis & & $-1,151$ \\
\hline
\end{tabular}

Fuente: elaboración propia en base al cuestionario de aprendizaje cooperativo en clase Nota. Análisis exploratorio de la percepción de los alumnos del tercer año del bachillerato general unificado sobre la aplicación del aprendizaje cooperativo.

Los datos se distribuyen de manera platicúrtica, con asimetría hacia la izquierda y desviación típica de 9,42. La suma total de las valoraciones otorgadas por los estudiantes, en cada ítem, varía en un rango de 30, siendo 57 la puntuación más baja y 87 la más alta. Esto permite afirmar que el nivel de aplicación del aprendizaje cooperativo tiene una tendencia no marcada de mediana igual a 72 y una media muestral de 72,96 que varía dentro del intervalo: [68,88; 77,03$]$, tal como se visualiza en la Tabla 4.

Los resultados muestran una aparente diferencia entre las percepciones de los estudiantes, por año, donde los alumnos de primero de bachillerato otorgan las puntuaciones más altas, que aparentemente disminuyen en forma gradual al aumentar los años. Esto se contrapone a las evaluaciones realizadas a los docentes, por lo que se requiere verificar si estas diferencias son significativas. 


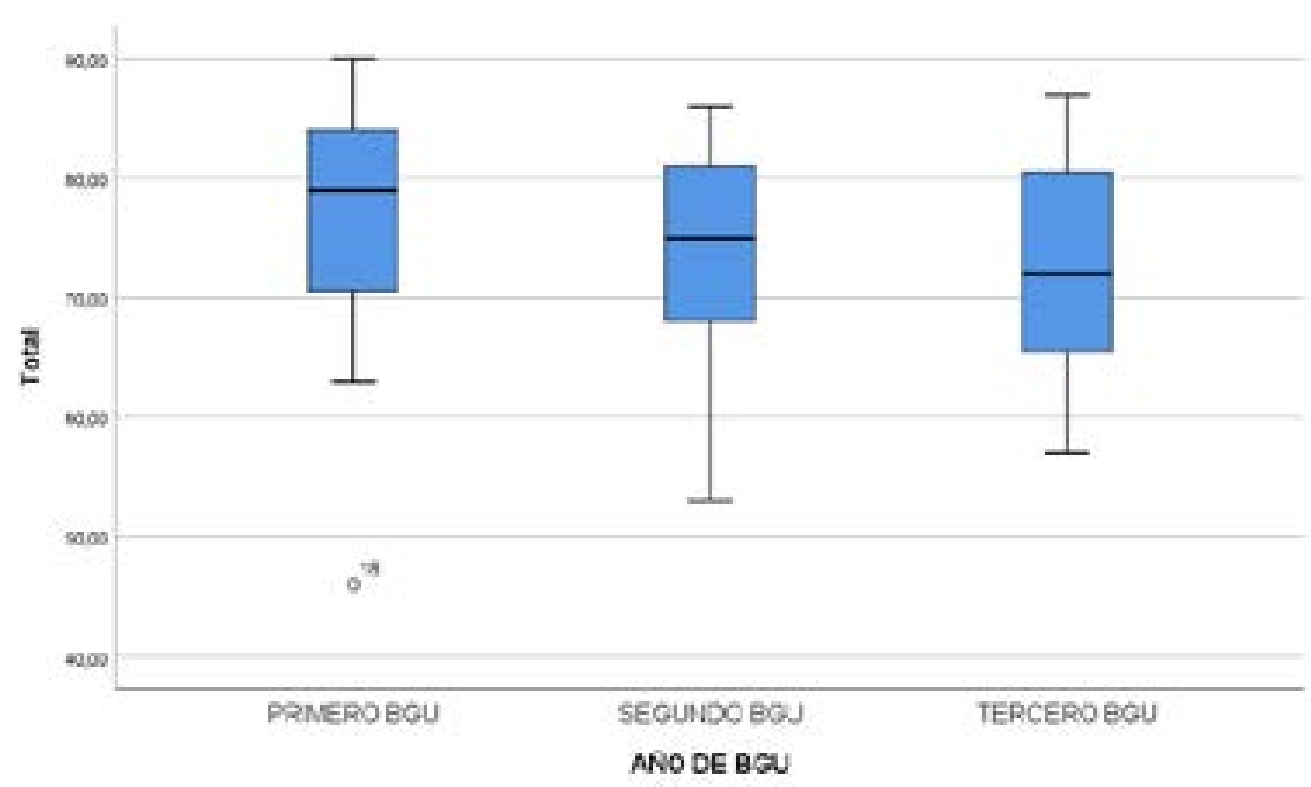

Figura 1. Diagrama comparativo, de cajas y bigotes-Aprendizaje cooperativo

Fuente: datos procesados en el Software SPSS

Nota. Diagrama comparativo del aprendizaje cooperativo del primer al tercer año del bachillerato general unificado.

La Figura 1 comparativa de cajas y bigotes permite apreciar, en primera instancia, que no existen diferencias significativas entre los tres grupos. Esta aseveración se comprueba con un análisis de varianza (se han probado previamente los supuestos de normalidad y homocedasticidad), cuyos resultados se muestran a continuación:

Tabla 5.

Prueba Fisher - diferencias significativas en el A.C.

\begin{tabular}{llllll}
\hline ANOVA & & & & & \\
\hline & $\begin{array}{l}\text { Suma de } \\
\text { cuadrados }\end{array}$ & gl & $\begin{array}{l}\text { Media } \\
\text { cuadrática }\end{array}$ & F & Sig. \\
\hline $\begin{array}{l}\text { Entre } \\
\text { grupos }\end{array}$ & 150,179 & 2 & 75,090 &, 798 &, 455 \\
\hline $\begin{array}{l}\text { Dentro de } \\
\text { grupos }\end{array}$ & 6214,110 & 66 & 94,153 & & \\
\hline Total & 6364,290 & 68 & & & \\
\hline
\end{tabular}

Fuente: elaboración propia en base a la Prueba Fisher

Nota. Prueba Fisher realizada para conocer las diferencias significativas del aprendizaje cooperativo aplicado en los alumnos pertenecientes al bachillerato.

El valor de prueba (Sig.) superior a la significancia del $5 \%$ muestra que la hipótesis es nula, por lo tanto, las diferencias no son significativas y se puede afirmar que en todos los años de bachillerato hay un mismo nivel de aprendizaje cooperativo durante la realización de actividades en el aula de clase. 
Con el fin de profundizar el estudio, se realiza un análisis de correlación entre las dimensiones que conforman el Cuestionario de Cooperación Activa en Clase (CCACL): Habilidades sociales, Procesamiento grupal, Interdependencia positiva, Interacción promotora y Responsabilidad individual.

Luego de probar, mediante el método de Kolmogorov - Smirnov, que la serie de datos no cumple con el supuesto de normalidad, se ha usado el coeficiente de correlación de Spearman para el análisis.

Tabla 6.

Análisis de correlación basado en las habilidades sociales

\begin{tabular}{llllll}
\hline & & $\begin{array}{l}\text { Pensamiento } \\
\text { grupal }\end{array}$ & $\begin{array}{l}\text { Independencia } \\
\text { positiva }\end{array}$ & $\begin{array}{l}\text { Interacción } \\
\text { promotora }\end{array}$ & $\begin{array}{l}\text { Responsabilidad } \\
\text { individual }\end{array}$ \\
\hline $\begin{array}{l}\text { Habilidades } \\
\text { sociales }\end{array}$ & $\begin{array}{l}\text { Coeficiente de } \\
\text { correlación }\end{array}$ &, $614^{\star \star}$ &, $503^{\star \star}$ &, $395^{\star \star}$ &, $454^{\star \star}$ \\
\cline { 2 - 6 } & Sig. (bilateral) &, 000 &, 000 &, 001 &, 000 \\
\cline { 2 - 6 } & $\mathrm{N}$ & 69 & 69 & 69 & 69 \\
\hline
\end{tabular}

Fuente: Cuestionario de Aprendizaje Cooperativo en Clase

Nota. Análisis de correlación de las habilidades sociales como son pensamiento grupal, independencia positiva, interacción promotora y responsabilidad individual.

En la Tabla 6 el valor de prueba (sig. Bilateral) inferior a 0,05 muestra que las habilidades sociales se correlacionan con todas las dimensiones de la cooperación activa en clase, siendo el procesamiento grupal y la interdependencia positiva las más relevantes.

Tabla 7.

Análisis de correlación basado en el pensamiento grupal

\begin{tabular}{llllll}
\hline & & $\begin{array}{l}\text { Habilidades } \\
\text { sociales }\end{array}$ & $\begin{array}{l}\text { Independencia } \\
\text { positiva }\end{array}$ & $\begin{array}{l}\text { Interacción } \\
\text { promotora }\end{array}$ & $\begin{array}{l}\text { Responsabilidad } \\
\text { individual }\end{array}$ \\
\hline $\begin{array}{l}\text { Pensamiento } \\
\text { grupal }\end{array}$ & $\begin{array}{l}\text { Coeficiente de } \\
\text { correlación }\end{array}$ &, $614^{\star \star}$ &, $445^{\star \star}$ &, $514^{\star \star}$ &, $536^{\star \star}$ \\
\cline { 2 - 6 } & Sig. (bilateral) &, 000 &, 000 &, 000 &, 000 \\
\cline { 2 - 6 } & $\mathrm{N}$ & 69 & 69 & 69 & 69 \\
\hline
\end{tabular}

Fuente: elaboración propia en base al cuestionario de aprendizaje cooperativo en clase

Nota. Análisis de correlación basado en el pensamiento grupal con el resto de las habilidades sociales valoradas.

El valor de prueba (sig. Bilateral) inferior a 0,05 muestra que el procesamiento grupal se correlaciona con todas las dimensiones de la cooperación activa en clase, siendo las habilidades sociales y la interacción promotora las más relevantes, así está reflejado en la Tabla 7. 
Tabla 8.

Análisis de correlación basado en la interdependencia

\begin{tabular}{llllll}
\hline & & $\begin{array}{l}\text { Habilidades } \\
\text { sociales }\end{array}$ & $\begin{array}{l}\text { Pensamiento } \\
\text { grupal }\end{array}$ & $\begin{array}{l}\text { Interacción } \\
\text { promotora }\end{array}$ & $\begin{array}{l}\text { Responsabilidad } \\
\text { individual }\end{array}$ \\
\hline $\begin{array}{l}\text { Independencia } \\
\text { positiva }\end{array}$ & $\begin{array}{l}\text { Coeficiente de } \\
\text { correlación }\end{array}$ &, $503^{\star \star}$ &, $445^{\star *}$ &, $561^{\star \star}$ &, $453^{\star \star}$ \\
\cline { 2 - 6 } & Sig. (bilateral) &, 000 &, 000 &, 000 &, 000 \\
\cline { 2 - 6 } & $\mathrm{N}$ & 69 & 69 & 69 & 69 \\
\hline
\end{tabular}

Fuente: elaboración propia en base al cuestionario de aprendizaje cooperativo en clase

Nota. Análisis de correlación basado en la independencia positiva con el resto de las habilidades sociales valoradas.

El valor de prueba (sig. Bilateral) inferior a 0,05 muestra que la interdependencia positiva se correlaciona con todas las dimensiones de la cooperación activa en clase, siendo las habilidades sociales y la interacción promotora las más relevantes (Tabla 8).

Tabla 9.

Análisis de correlación basado en la interacción promotora

\begin{tabular}{llllll}
\hline & & $\begin{array}{l}\text { Habilidades } \\
\text { sociales }\end{array}$ & $\begin{array}{l}\text { Pensamiento } \\
\text { grupal }\end{array}$ & $\begin{array}{l}\text { Independencia } \\
\text { positiva }\end{array}$ & $\begin{array}{l}\text { Responsabilidad } \\
\text { individual }\end{array}$ \\
\hline $\begin{array}{l}\text { Interacción } \\
\text { promotora }\end{array}$ & $\begin{array}{l}\text { Coeficiente de } \\
\text { correlación }\end{array}$ &, $395^{\star \star}$ &, $514^{\star \star}$ &, $561^{\star \star}$ &, $624^{\star \star}$ \\
\cline { 2 - 6 } & Sig. (bilateral) &, 001 &, 000 &, 000 &, 000 \\
\cline { 2 - 6 } & $\mathrm{N}$ & 69 & 69 & 69 & 69 \\
\hline
\end{tabular}

Fuente: elaboración propia en base al cuestionario de aprendizaje cooperativo en clase

Nota. Análisis de correlación basado en la interacción promotora con el resto de las habilidades sociales valoradas.

El valor de prueba (sig. Bilateral) inferior a 0,05 muestra que la interacción promotora (ver Tabla 9) se correlaciona con todas las dimensiones de la cooperación activa en clase, siendo la responsabilidad individual y la interdependencia positiva las más relevantes.

Tabla 10.

Análisis de correlación basado en la responsabilidad individual

\begin{tabular}{llllll}
\hline & & $\begin{array}{l}\text { Habilidades } \\
\text { sociales }\end{array}$ & $\begin{array}{l}\text { Pensamiento } \\
\text { grupal }\end{array}$ & $\begin{array}{l}\text { Independencia } \\
\text { positiva }\end{array}$ & $\begin{array}{l}\text { Interacción } \\
\text { promotora }\end{array}$ \\
\hline $\begin{array}{l}\text { Responsabilidad } \\
\text { individual }\end{array}$ & $\begin{array}{l}\text { Coeficiente de } \\
\text { correlación }\end{array}$ &, $454^{\star *}$ &, $536^{* *}$ &, $453^{\star *}$ &, $624^{\star *}$ \\
\cline { 2 - 6 } & Sig. (bilateral) &, 000 &, 000 &, 000 &, 000 \\
\cline { 2 - 6 } & $\mathrm{N}$ & 69 & 69 & 69 & 69 \\
\hline
\end{tabular}

Fuente: elaboración propia en base al cuestionario de aprendizaje cooperativo en clase

Nota. Análisis de correlación basado en la responsabilidad individual con el resto de las habilidades sociales valoradas. El valor de prueba (sig. Bilateral) inferior a 0,05 muestra que la responsabilidad individual (ver Tabla 10) se correlaciona con todas las dimensiones de la cooperación activa en clase, siendo la interacción promotora y el procesamiento grupal las más relevantes. 
Finalmente, utilizando el software NVivo12 Plus se ha realizado un análisis cualitativo de las entrevistas realizadas a los docentes. El método escogido es el análisis de frecuencia de palabras, del cual se extrae lo siguiente:

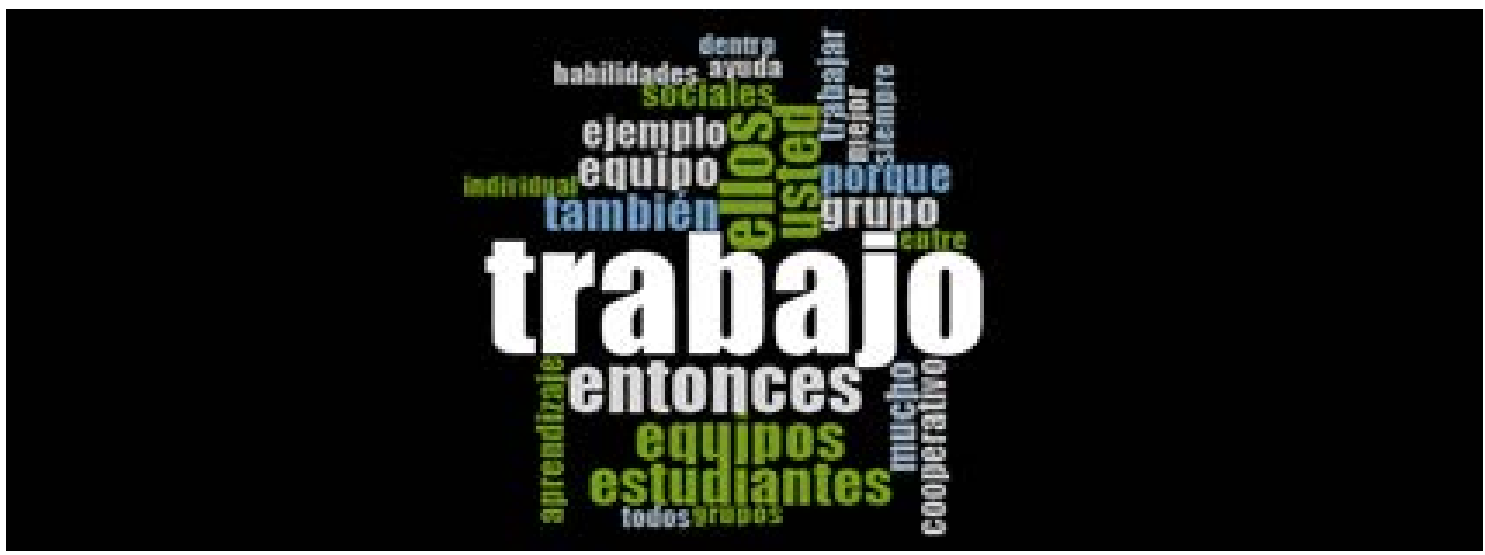

Figura 2. Análisis cualitativo - Marca de nube con resultados de entrevista

Fuente: elaboración propia

Nota. Marca de nube con resultados de entrevista realizada a los docentes.

En la Figura 2 se identifican las 25 palabras más frecuentes (de mínimo 5 letras), usadas por los docentes durante las entrevistas. De estas palabras, se descartan las que no son relevantes (por ser de conexión) para el estudio. Por lo tanto, los términos más relevantes son: "trabajo", "equipos", "grupos"; "cooperativo"; "aprendizaje"; "estudiantes"; "ejemplo"; "habilidades"; "sociales" y "ayuda".

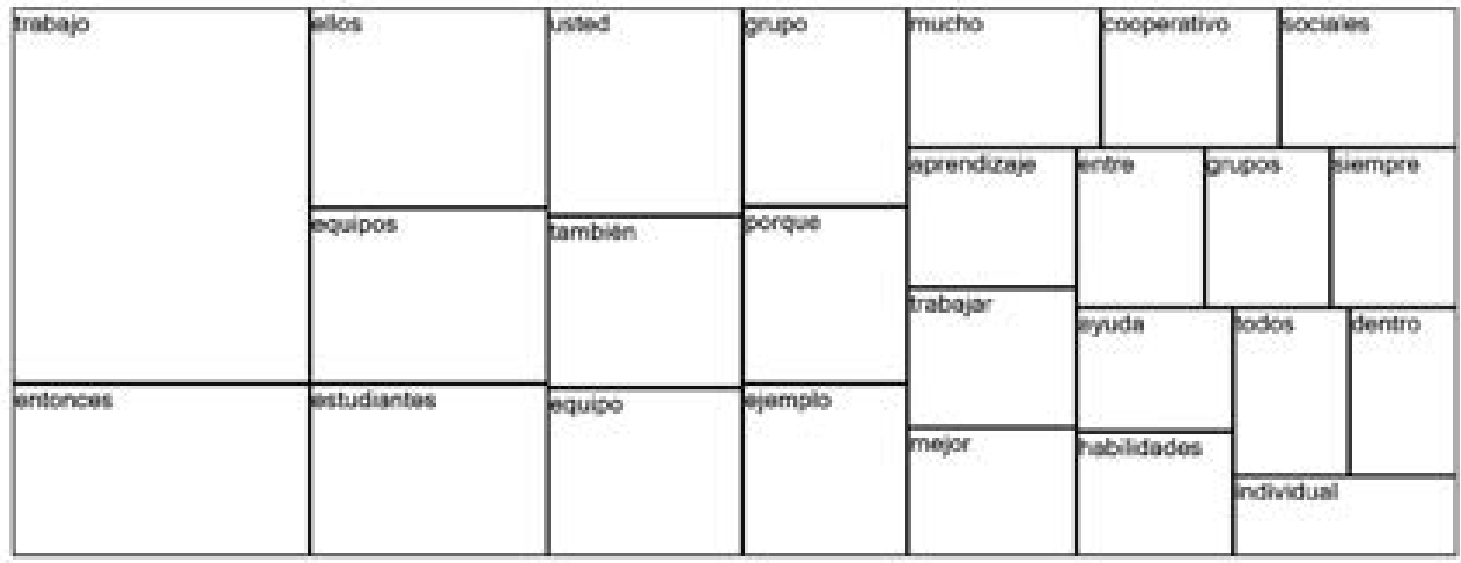

Figura 3. Análisis cualitativo - Mapa ramificado - Con resultados de entrevista

Fuente: NVIVO 10

Nota. Como resultado de la entrevista se realizó un análisis cualitativo y se efectuó mapa ramificado

El mapa ramificado (Figura 3) muestra las conexiones entre las palabras: trabajo y entonces con los demás términos frecuentes. Tomando en cuenta solo lo relevante, se puede apreciar que los entrevistados coinciden en que el trabajo cooperativo está basado en la formación de grupos o 
equipos de aprendizaje, que desarrollan habilidades sociales e individuales mediante la realización de actividades guiadas con ejemplos y ayuda del docente.

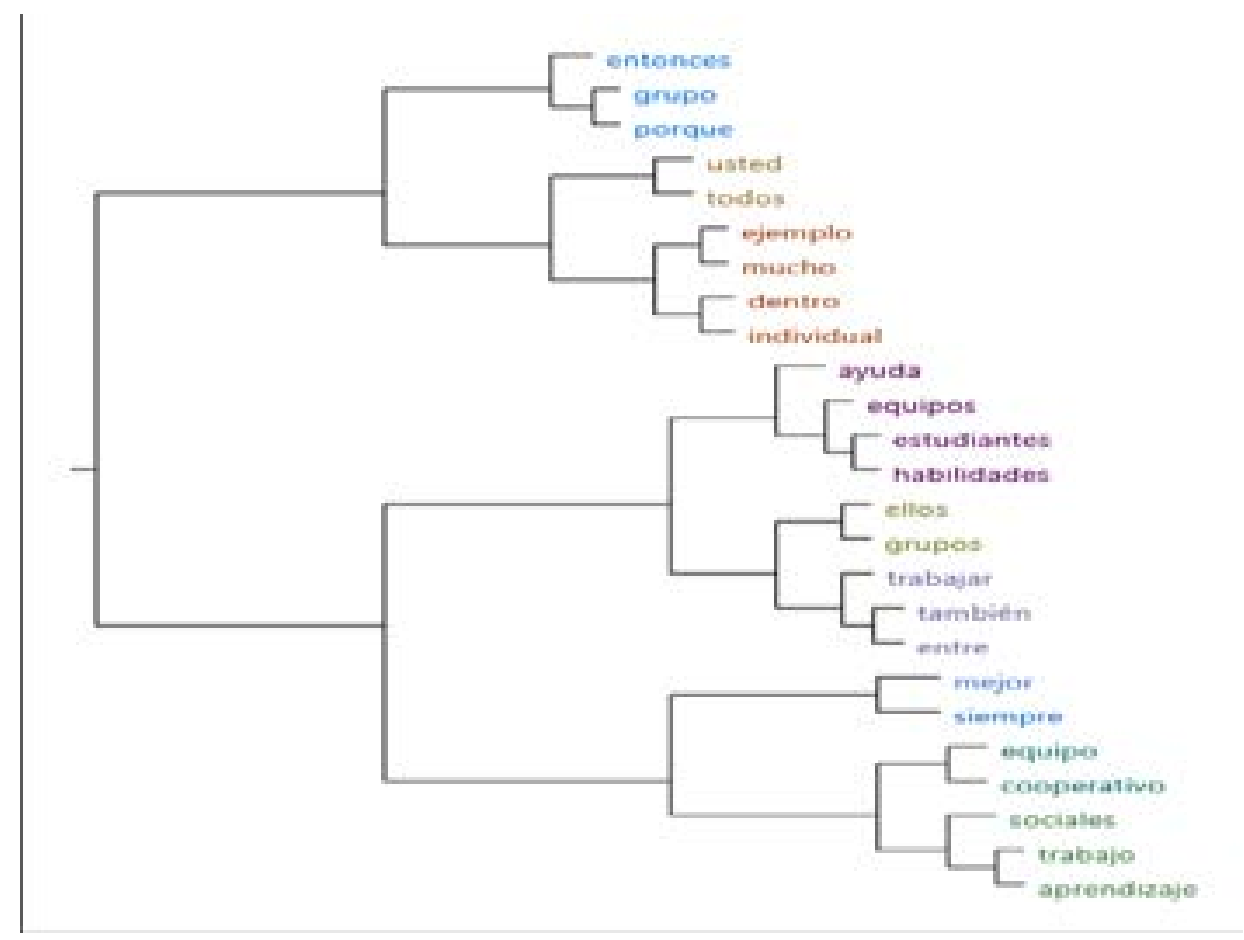

Figura 4. Análisis de conglomerados basado en resultados de entrevista.

Fuente: NVIVO 10 / Nota. Como resultado de la entrevista se realizó un análisis de conglomerados

La Figura 4 muestra la presencia de varios conglomerados, formados en función de las relaciones entre las palabras más frecuentes. Aquí se puede apreciar que los términos: trabajo y aprendizaje están ligados a sociales, equipo y cooperativo. Otro conglomerado importante es el que está formado por: habilidades, estudiantes, equipos y ayuda.

\section{Resultados}

Al realizar un análisis de los resultados cuantitativos y cualitativos referente al aprendizaje cooperativo, es notorio darse cuenta que los docentes de las asignaturas de Matemática y Lengua y Literatura no promueven el aprendizaje cooperativo y desconocen de la forma de trabajar en clase con este tipo de aprendizaje innovador, a esta práctica decadente, se contraponen Doporto y Rodríguez (2015); Seijo, López, C., López, M. y Virseda (2015) donde dan a conocer que el docente debe estar formado en este tipo de aprendizaje desde la manera de planificar, la forma de enseñar, el modo de agrupar e interactuar, el interés por adoptar planes de mejora, realizar ajustes y modificaciones en el currículo y, que deben estar conscientes que este tipo de aprendizaje fortalece el desarrollo personal y las habilidades sociales en los estudiantes de bachillerato.

En la aplicación del Cuestionario de Cooperación Activa en Clase (CCACL) los resultados en primera instancia mostraron mayor tendencia de aplicación en los estudiantes de Primero de 
Bachillerato y que gradualmente iba decayendo en los años de Segundo y Tercero de Bachillerato. Y que al realizar un análisis más profundo se concluye que en todos los años existe un mismo nivel de aplicación de aprendizaje cooperativo. A esta idea de aplicación de aprendizaje cooperativo en las aulas se une Deutsch (1949), Hulten y De Vries (1976) al dar a conocer que, en un aula cooperativa, los estudiantes asisten con puntualidad, se sienten a gusto y muy motivados por sus compañeros, reciben méritos y oportunidades para ser mejor cada día.

Con respecto a los componentes esenciales del aprendizaje cooperativo que son: las habilidades sociales, interdependencia positiva, interacción social, y el procesamiento grupal; en el análisis de correlación, muestra que todos estos componentes se correlacionan entre sí, pero en mayor medida las habilidades sociales con el procesamiento grupal y la interdependencia positiva; así también el procesamiento grupal con las habilidades sociales e interacción promotora; de la misma manera la interdependencia positiva tiene mayor grado de correlación con las habilidades sociales y la interacción promotora; por otro lado la interacción promotora guarda estrecha relación con la responsabilidad individual e interdependencia positiva; y finalmente la responsabilidad individual con la interacción promotora y el procesamiento grupal.

Para corroborar lo expuesto anteriormente Johnson, D., Johnson, R., y Holubec (1999) mencionan a estos cinco componentes como esenciales en el aprendizaje cooperativo, pues en base a estos los estudiantes interactúan entre todos, cada miembro del equipo es responsable de su aporte, se motivan mutuamente, trabajan por un bien común, toman decisiones de manera consensuada, respetan, escuchan y toleran.

En los resultados obtenidos en la entrevista realizada a los docentes de las áreas básicas de bachillerato concuerdan en que el trabajo cooperativo está fundamentado en la formación de grupos o equipos de aprendizaje, que desarrollan habilidades sociales e individuales mediante la realización de actividades guiadas con ejemplos y ayuda del docente. Así también lo dan a conocer Gómez y Gómez (2016) y Cifuentes y Meseguer (2015) que el aplicar el aprendizaje cooperativo promueve las relaciones sociales, la interrelación entre compañeros y docentes, la creatividad, la responsabilidad y autonomía.

\section{Conclusiones}

El aprendizaje cooperativo se constituye en una estrategia pedagógica innovadora que rompe esquemas tradicionales y abre las puertas a la aplicación de un aprendizaje compartido, a una educación desde la participación social y que aporta sustancialmente al ámbito de la solidaridad como uno de los ejes primordiales en el perfil de salida del bachiller ecuatoriano.

Los componentes esenciales del aprendizaje cooperativo son las habilidades sociales, la interdependencia positiva, la interacción social, y el procesamiento grupal; que se constituyen en aspectos primordiales para lograr aprendizajes significativos en equipo, empatía y buenas relaciones entre los integrantes, resultados eficientes y gran ayuda y colaboración entre los integrantes. Así también estos componentes esenciales se correlacionan unos con otros y forman un engranaje fluido y eficiente al momento de trabajar de manera cooperativa. De la misma manera requieren de una 
acción disciplinada de docentes y estudiantes, de procesos rigurosos, muy bien establecidos y muy bien cumplidos.

Los profesionales de las asignaturas relacionadas a las ciencias exactas tienden a aplicar en menor medida el aprendizaje cooperativo, mientras que los profesores de las materias de carácter social tienden a desarrollar en mayor grado esta estrategia didáctica.

La participación social generada por la aplicación del aprendizaje cooperativo no está ligada al año de bachillerato, puesto que existen otros factores que motivan el desarrollo de la participación social en los estudiantes.

Para lograr resultados favorables en la aplicación del aprendizaje cooperativo se requiere de tiempo, recursos, capacitación docente, planeación, organización, ejecución, control y evaluación.

El aprendizaje cooperativo en bachillerato en muchos de los casos es aplicado de manera empírica, pues los docentes no tienen un dominio del tema, desconocen de las estrategias de enseñanza-aprendizaje que se maneja en este tipo de aprendizaje; lo cual desemboca en la aplicación de un A.C. improvisado, tedioso, poco objetivo. Esto hace pensar que el aprendizaje cooperativo no se constituye en una estrategia eficiente en la educación, cuando en la aplicabilidad de manera correcta y eficiente resulta ser todo lo contrario.

En relación a los resultados obtenidos se sugiere que el aprendizaje cooperativo debe ser introducido en las aulas como si se tratara de una asignatura más, debido a la complejidad que resulta en manejarlo, su amplio contenido en materia y aplicación, el involucramiento total tanto de docentes como de estudiantes, y el trabajo en ejes transversales como la solidaridad, la tolerancia, el respeto, la cooperación, el amor, el compromiso, la puntualidad, la perseverancia. 


\section{Referencias bibliográficas}

Bell, N., Grossen, M., y Perret-Clermont, A. N. (1985). Sociocognitive conflict and intellectual growth. New Directions for Child and Adolescent Development, 1985(29), 41-54.

Berbena, M. A. Z., Sierra, M. D. L. D. V., y Vivero, M. D. C. V. (2017). Inteligencia emocional y habilidades sociales en adolescentes con alta aceptación social. Electronic Journal of Research in Education Psychology, 6(15), 321-339.

Cifuentes Férez, P., y Meseguer Cutillas, P. (2015). Trabajo en equipo frente a trabajo individual: ventajas del aprendizaje cooperativo en el aula de traducción. Tonos Digital, 28(0).

Coleman, J. S. (1961). The adolescent society: The social life of the teenager and its impact on education.

Deutsch, M. (1949). A theory of co-operation and competition. Human relations, 2(2), 129-152.

Doporto, S. L., y Rodríguez, M. M. C. (2015). El Aprendizaje Cooperativo, un camino hacia la inclusión educativa. Revista complutense de Educación, 27(3), 1085-1101.

Fernández-Rio, J., Cecchini, J. A., Méndez-Giménez, A., Méndez-Alonso, D., y Prieto, J. A. (2017). Diseño y validación de un cuestionario de medición del aprendizaje cooperativo en contextos educativos. Anales de Psicología/Annals of Psychology, 33(3), 680-688.

Gómez, M. P., y Gómez, Á. H. (2016). Experiencia docente acerca del uso didáctico del aprendizaje cooperativo y el trabajo de campo en el estudio del fenómeno de influencia social. Revista electrónica interuniversitaria de formación del profesorado, 19(2), 331-346.

Hernández - Sampieri, R., Fernández, C., \& Baptista, P. (2014). Metodología de la investgación (Sexta edic). México D.F: McGRW HILL.

Hernández Sampieri, R., \& Fernández Collao, C. (2016). Libro Metodología de la investigación SAMPIERI. Libro Metodología de la investigación SAMPIERI.: McGraw Hill education.

Hulten, B. H., y DeVries, D. L. (1976). Team Competition and Group Practice: Effects on Student Achievement and Attitudes. Report No. 212.

Johnson, D. W., Johnson, R. T., y Holubec, E. J. (1999). El aprendizaje cooperativo en el aula.

López, G., y Acuña, S. (2018). Aprendizaje cooperativo en el aula. Inventio, la génesis de la cultura universitaria en Morelos, 7(14), 29-38.

Martín, R. B., Martín, J. J. B., y Mayo, P. G. (2014). El papel crítico y reflexivo del profesorado ante el aprendizaje 
cooperativo. EmásF: revista digital de educación física, (29), 8-18.

MINEDUC (2016). Currículo de los niveles de educación obligatoria. Quito: Ministerio de Educación. Recuperado de: https://educacion.gob.ec/wp-content/uploads/downloads/2016/08/Curriculov2.pdf.

Pinos-Morales, G., Ayala-Gavilanes, D., \& Bonilla-Jurado, D. (2019). Desarrollo del pensamiento lógico-matemático a través de juegos populares y tradicionales en niños de educación inicial. Revista Científica Ciencia y tecnología, 18(19).

Román, A. M. G. C., y de la Torre, E. H. (2017). El aprendizaje cooperativo como estrategia para la inclusión del alumnado con tea/as en el aula ordinaria. Revista de Educación Inclusiva, 9(2-bis).

Seijo, J. C. T., López, C. M., López, M. L. P., y Virseda, C. M. (2015). Formación del profesorado en aprendizaje cooperativo y alumnos con altas capacidades: un enfoque inclusivo. Revista latinoamericana de educación inclusiva, 9(2), 91-110.

Slavin, R. E., y Johnson, R. T. (1999). Aprendizaje cooperativo: teoría, investigación y práctica. Buenos Aires: Aique.

UNESCO (2011). Office Santiago and Regional Bureau for Education in Latin America and the Caribbean. Spain. Ministerio de Educación. Recuperado de: https://unesdoc.unesco.org/ark:/48223/pf0000193130?posInSet=1yqueryld=441cf6d6-8898-47fe-82a6-135d0870398f

Vygotsky, L. (1978). Interaction between learning and development. Readings on the development of children, 23(3), 34-41.

Vygotsky, L. S. (1979) El desarrollo de los procesos psicológicos superiores. Buenos Aires: Grijalbo. 
Copyright (c) 2020 Wilmer Rubén Paredes León y Gerardo Ramos Serpa

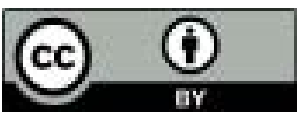

Este texto está protegido bajo una licencia internacional Creative Commons 4.0.

Usted es libre para Compartir - copiar y redistribuir el material en cualquier medio o formato - y Adaptar el documento - remezclar, transformar y crear a partir del material-para cualquier propósito, incluso para fines comerciales, siempre que cumpla las condiciones de Atribución. Usted debe dar crédito a la obra original de manera adecuada, proporcionar un enlace a la licencia, e indicar si se han realizado cambios. Puede hacerlo en cualquier forma razonable, pero no de forma tal que sugiera que tiene el apoyo del licenciante o lo recibe por el uso que hace de la obra.

Resumen de licencia - Texto completo de la licencia 